STUDI

FRANCESI

\section{Studi Francesi}

Rivista quadrimestrale fondata da Franco Simone

159 (LIII | III) | 2009

Varia

\title{
Aa. Vv., Correspondance de Pierre Bayle. Tome V (août 1684- fin juillet 1685), Lettres 309-450 et t. VI (août 1685-fin juin 1686), Lettres 451-587
}

\section{Franco Piva}

\section{(2) OpenEdition}

\section{Journals}

Edizione digitale

URL: http://journals.openedition.org/studifrancesi/7566

DOI: $10.4000 /$ studifrancesi.7566

ISSN: 2421-5856

\section{Editore}

Rosenberg \& Sellier

\section{Edizione cartacea}

Data di pubblicazione: 1 décembre 2009

Paginazione: 622-623

ISSN: 0039-2944

\section{Notizia bibliografica digitale}

Franco Piva, «Aa. Vv., Correspondance de Pierre Bayle. Tome V (août 1684- fin juillet 1685), Lettres 309-450 et t. VI (août 1685-fin juin 1686), Lettres 451-587», Studi Francesi [Online], 159 (LIII | III) | 2009, online dal 30 novembre 2015, consultato il 09 janvier 2021. URL: http://journals.openedition.org/ studifrancesi/7566 ; DOI: https://doi.org/10.4000/studifrancesi.7566

Questo documento è stato generato automaticamente il 9 janvier 2021.

\section{cc) (†)}

Studi Francesi è distribuita con Licenza Creative Commons Attribuzione - Non commerciale - Non opere derivate 4.0 Internazionale. 


\title{
Aa. Vv., Correspondance de Pierre Bayle. Tome V (août 1684- fin juillet 1685), Lettres 309-450 et t. VI (août 1685-fin juin 1686), Lettres 451-587
}

\author{
Franco Piva
}

\section{NOTIZIA}

Correspondance de Pierre Bayle. Tome V (août 1684- fin juillet 1685), Lettres 309-450 et t. VI (août 1685-fin juin 1686), Lettres 451-587. Publiées et annotées par Elisabeth LABROUSSE et Anthony MCKENNA, Laurence BERGON, Hubert BOST, Wiep VAN BUNGE, Edward JAMES, Annie LEROUX, Caroline VERDIER, Fabienne VIAL-BONNACCI. Avec la collaboration de Eric-Olivier LOCHARD, Oxford, Voltaire Foundation, 2007 et 2008, pp. XXIII+513 et XXIV+508.

1 I due volumi sono dedicati alla memoria di Elisabeth Labrousse, la grande studiosa di Bayle, da poco scomparsa, che con Anthony McKenna è all'origine della grande idea di riunire in un'edizione critica accuratamente annotata la corrispondenza completa di uno degli uomini che a mano a mano che gli anni passano e che gli studi si moltiplicano, appare come una delle figure chiave di quel complesso periodo di trapasso tra Sei e Settecento che Pierre Bayle ha vissuto con straordinaria intensità e partecipazione e che ha saputo interpretare come pochi altri al suo tempo. L'impresa, iniziata alcuni anni orsono, procede spedita non soltanto grazie all'importante lavoro preparatorio predisposto da Elisabeth Labrousse e da Anthony McKenna, ma anche grazie alla squadra di studiosi che con loro ha collaborato fin dall'inizio all'impresa e che saprà certamente supplire al meglio la scomparsa della prima ideatrice dell'edizione in corso.

2 I due volumi che presentiamo in questa scheda sono importanti ed interessanti per diversi motivi. Il primo dei due, il quinto dell'edizione completa, comprende le lettere 
scritte da Pierre Bayle o a lui indirizzate in un momento particolarmente delicato della sua vita di uomo e di letterato: da un lato, Bayle ha appena appreso la morte dell'amato fratello minore; dall'altro, ha da poco lanciato la grande impresa delle Nouvelles de la République des Lettres; le lettere della prima parte del volume hanno quindi a che fare innanzitutto con questi due avvenimenti; accanto alle condoglianze per la perdita del fratello, Bayle riceve i complimenti per il successo che la rivista sta incontrando presso le "gens de lettres" per cui Bayle l'ha concepita. Le lettere che seguono ci consentono di scoprire l'ampia rete di conoscenze che permettono a Bayle di essere al corrente di tutto quanto d'importante succede attorno a lui, e dei libri che si pubblicano e dei quali vale la pena dar conto. Le principali conoscenze di Bayle si situano, evidentemente, nel mondo del Rifugio olandese, ma a mano a mano che l'impresa giornalistica prosegue, e che le Nouvelles si impongono all'attenzione dei lettori, il mondo, e i corrispondenti di Bayle, si allargano all'ambiente protestante di altri paesi, con qualche aggancio anche nel mondo cattolico, dentro al quale non manca chi apprezza la sua dirittura morale e la sua scienza.

3 Una parte importante della corrispondenza di Bayle relativa a questo periodo riguarda - né poteva essere diversamente - le persecuzioni di cui sono sempre più spesso vittime gli ugonotti francesi e le opere della controversia che li oppone ai cattolici, e di cui Bayle dà, seppure con prudenza, conto. Bayle si dimostra al corrente sia delle dragonnades che imperversano nelle Cevennes, nel Dauphiné e nel Vivarais sia dei tumulti scoppiati, fa osservare Anthony McKenna nella sua lucida ricostruzione del clima dei mesi che precedettero la revoca dell'Editto di Nantes, «à la suite du projet que Claude Brousson a élaboré en juillet 1683, qui consiste à organiser des cultes sur les "masures" des temples abattus», i quali provocarono ulteriori e più dure repressioni, accelerando forse l'editto di Fontainebleau, le cui conseguenze sia pratiche che ideologiche occupano quasi tutto il volume seguente. Da un lato con gli echi della fuga in massa degli Ugonotti sorpresi, malgrado tutto, dalla Revoca, ed in particolare da quella dei loro pastori, ai quali per aver salva la vita sono date poche settimane, quando non addirittura poche ore per lasciare il Paese che li aveva visti nascere. Dall'altro, con gli echi delle polemiche che l'editto provocò non solo tra i protestanti, tra i quali emerge per la virulenza dei suoi attacchi la figura, già nota, di Jurieu, ma anche tra alcuni cattolici, che non riuscirono a nascondere il loro disappunto. Del resto gli avvenimenti di quei mesi colpiscono Bayle doppiamente in quanto al disagio per la debolezza di Luigi XIV di fronte alle pressioni cattoliche, s'aggiunge il dolore per la perdita del fratello Jacob, da poco succeduto al vecchio padre nelle funzioni di pastore e della cui morte Bayle si sente in qualche modo anche se involontariamente, responsabile.

4 Le lettere contenute in questo volume della Correspondance lasciano però anche vedere lo svilupparsi dell'attività di Bayle nel campo intellettuale sia per quanto riguarda le Nouvelles de la République des Lettres che conoscono un successo inatteso non solo negli ambienti protestanti ma anche in Francia, sia per quanto riguarda la polemica innescata dalla revoca dell'editto di Nantes alla quale Bayle partecipa, tra l'altro, con il suo celebre Commentaire nel quale riprese e sviluppò le teorie del suo amico e protettore Adrian Paets.

Quello di cui testimoniano le lettere contenute in questo volume è dunque un periodo molto doloroso per Pierre Bayle sul piano personale, ma anche molto intenso e fecondo sul piano intellettuale. Il giornalista prosegue la sua conquista della République des 
Lettres ed il filosofo prepara alcune delle sue opere più importanti. Nella lettura delle lettere e nella ricostruzione degli avvenimenti all'interno dei quali ciascuna si situa, il lettore è grandemente aiutato dalla ricca ed accurata annotazione che accompagna ogni singola lettera; così come molto utile è anche l'«Index des noms de personnes» che chiude ogni volume. Davvero un'encomiabile impresa quindi che non mancherà di essere di notevole aiuto a quanti vorranno conoscere più da vicino questo uomo schivo ma essenziale alla comprensione di quell'epoca complessa che è passata alla Storia come quella della «crise de la conscience européenne» e di cui Bayle è stato non solo uno dei protagonisti di maggiore spicco ma anche un interprete eccezionalmente lucido. 